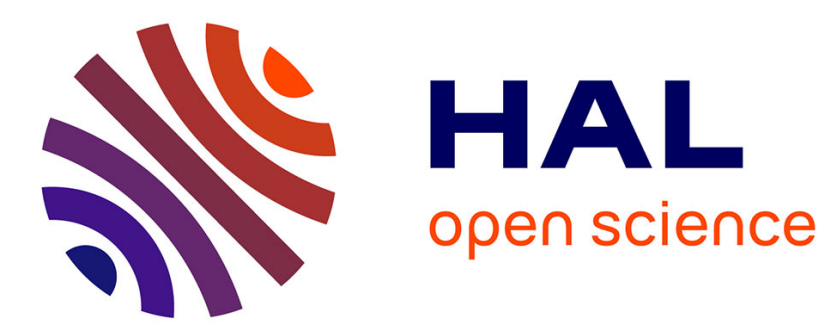

\title{
Extended Space Shift Keying Modulation With Different Receiver Strategies
}

\author{
Ali Mokh, Maryline Hélard, Matthieu Crussière
}

\section{To cite this version:}

Ali Mokh, Maryline Hélard, Matthieu Crussière. Extended Space Shift Keying Modulation With Different Receiver Strategies. International conference on telecommunication, Apr 2019, hanoi, Vietnam. hal-01976078

\section{HAL Id: hal-01976078 \\ https://hal.science/hal-01976078}

Submitted on 9 Jan 2019

HAL is a multi-disciplinary open access archive for the deposit and dissemination of scientific research documents, whether they are published or not. The documents may come from teaching and research institutions in France or abroad, or from public or private research centers.
L'archive ouverte pluridisciplinaire HAL, est destinée au dépôt et à la diffusion de documents scientifiques de niveau recherche, publiés ou non, émanant des établissements d'enseignement et de recherche français ou étrangers, des laboratoires publics ou privés. 


\title{
Extended Space Shift Keying Modulation With Different Receiver Strategies
}

\author{
Ali Mokh, Maryline Hélard and Matthieu Crussière \\ Univ Rennes, INSA Rennes, IETR, CNRS, UMR 6164, F-35000 Rennes
}

\begin{abstract}
Space Shift Keying is a MIMO transmission schemes that has been recently studied as promising solution for low computational complexity devices. Such scheme is based on the concept of spatial modulations (SM) where the information is mapped to the index of the transmit (resp. the receive) antenna. When only one antenna is used per symbol duration, an SSK symbol is able to transmit base $2 \mathrm{log}$ of the number of transmit antenna $\left(N_{t}\right)$. In this paper, we introduce an extended version of the conventional SSK scheme, refereed to as Extended SSK (ESSK). The proposed technique allows the overall spectral efficiency to be increased such that the total amount of bits reaches $N_{t}$ instead of $\log _{2}\left(N_{t}\right)$ in conventional approaches. In ESSK, one so-called spatial symbol corresponds to a set of bits that are mapped to a set of transmit antennas of variable size that are activated at each time symbol. The performance of ESSK is analyzed in this paper and validated through simulations.
\end{abstract}

Index Terms-MIMO,Spatial Modulation, Space Shift Keying, Zero Forcing, Equalization

\section{INTRODUCTION}

Spatial modulation (SM) schemes based on Multiple-input and multiple-output (MIMO) wireless systems, appeared in the early 2000s, consisting in exploiting the index of the transmit or receive antennas to transmit additional information bits. First proposed SM schemes concerned open loop systems because requiring no channel state information (CSI) at the transmitter side. They can be called TSM for transmit SM schemes; the spatial information is carried out by the index of the transmit antennas. One popular of these TSM schemes is Space Shift Keying (SSK) scheme [1] whose main principle is to take advantage of the various propagation characteristics associated to the different antennas of the system [2]. The signal processing at the receiver can also be simplified as data are transmitted through a single spatial stream in SMMIMO systems [3]. A generalized technique for SSK, further referred to as Generalized Space Shift Keying (GSSK), was proposed in [4]. Such a scheme overcomes the constraint in SM that the number of transmit antennas $N_{t}$ has to be a power of two, by activating during each symbol duration a subset of $N_{a}$ TAs out of the $N_{t}$ available at the transmit antenna array, to increase the overall SE.

The same concept of SM-MIMO has been applied to the receiver, called Receive-Spatial Modulation (RSM) [5] [6], or Receive Antenna Shift Keying (RASK) [7][8], where the index of the targeted receive antenna (RA) carries the additional spatial information, using a pre-processing technique to target the RAs. A generalization of the RSM principle, further referred to as GPSM (Generalised Pre-coding aided
Spatial Modulation), is proposed in [9] where the transmit antennas concentrate the signal energy towards a fixed and constant number of RAs to increase the SE. Opposed to conventional SM where a subset of RF chains is deployed, here all TAs and RAs are active. Finally, an extended model of RASK is introduced in [10], referred to as ERASK, where all possible combinations of targeted RAs are used, even that one where zero RA is targeted, i.e. no signal is emitted. Among other candidates, this last scheme offers the highest spectral efficiency at the level of the spatial bits, i.e. equal to the number of receive antennas [11][12]. With same analogy at the transmitter, Extended SSK (ESSK) has been proposed in [13] for transmit SM concept where the number of active antenna is variable, giving a SE equal to $N_{t}$. In this paper, we evaluated the performance of ESSK scheme using different detection strategies. The Maximum Likelihood detector that can be carried out at the receiver side is first proposed and studied as well as the expected theoretical performance. Also, we compare its performance to the performance of the system when Zero-Forcing equalization is performed at the receiver before the detection. At the end of this paper, we demonstrate the duality of ESSK with the ERASK scheme.

\section{SyStem MODEL AND Block DiagRAM}

In this part, we first set up the model for a communication system making use of the ESSK scheme. Then, we describe the block diagram of ESSK transmission.

\section{A. System Model}

MIMO system with $N_{t}$ transmit antennas and $N_{r}$ receive antennas is considered. Assuming a flat fading channel between the transmitter and the receiver, the receive signal vector can be written as:

$$
\mathbf{y}=\sqrt{\frac{2}{N_{t}}} \mathbf{H} . \mathbf{x}+\mathbf{n}
$$

where $\mathbf{H} \in \mathbb{C}^{N_{r} \times N_{t}}$ is the MIMO channel matrix with element $h_{j, i}$ representing the complex channel coefficient between the $i$-th transmission antenna, denoted by $T_{i}$, and the $j$-th receiving antenna, denoted by $R_{j} . \mathbf{y} \in \mathbb{C}^{N_{r} \times 1}$ is the vector of the received signals at all the receive antennas, $\mathbf{x} \in \mathbb{N}^{N_{t} \times 1}$ is the vector of the spatial symbol that will be transmitted by all the transmit antennas, and $\mathbf{n} \in \mathbb{C}^{N_{r} \times 1}$ is the vector of additive white Gaussian noise (AWGN) samples $\eta_{j}$ such that $\eta_{j} \sim \mathcal{C N}\left(0, \sigma_{n}^{2}\right)$. 


\section{B. Principle Block Diagram}

The ESSK scheme is built on the SSK concept at the transmitter where the number of active antennas $N_{a}$ is variable during each time symbol $T_{s}\left(0 \leq N_{a} \leq N_{t}\right)$. As for ERASK [10], $N_{a}$ can take all possible values between 0 and $N_{r}$ depending on the useful information to transmit so that the number of possible spatial symbols is $M=2^{N_{t}}$ providing the transmission of $N_{t}$ bits per spatial symbol.

\begin{tabular}{|c||c|c|c|c|}
\hline Symbol & 00 & 01 & 10 & 11 \\
\hline Active Antennas & $\mathrm{x}$ & $T_{2}$ & $T_{1}$ & $T_{1}, T_{2}$ \\
\hline \multicolumn{5}{|c}{ Table I }
\end{tabular}

ESSK SPATIAL MAPPING FOR $N_{t}=2$

Table I provides an illustration of the ESSK symbol mapping with 2 transmit antennas, and is able to transmit 2 bits/symbol. The block diagram of the ESSK system is depicted in Figure 1. A group of $m=N_{t}$ bits is mapped to a spatial symbol $\mathbf{x} \in \mathbb{C}^{N_{t} \times 1}$ which is written as:

$$
\mathbf{x}=\left[\begin{array}{llll}
x_{1} & x_{2} & \ldots & x_{N_{t}}
\end{array}\right]^{T} \text { where } x_{i} \in\{0,1\} .
$$

The value taken by each $x_{i}$ entry determines the set of active transmit antennas such that:

$$
x_{i}= \begin{cases}0, & \text { if } T_{i} \text { is not active } \\ 1, & \text { if } T_{i} \text { is active }\end{cases}
$$

The spatial symbol vector is normalized by a factor $f=\sqrt{\frac{2}{N_{t}}}$ before being emitted, used to guarantee that the average total transmit power $\bar{P}_{t}$ is equal to 1 .

\section{Detection Strategies}

\section{A. Maximum Likelihood (ML)}

1) $M L$ detection equation: At the receiver, a number $N_{r}$ of receive antennas detect the signal. From equation (1), if we are sending the spatial symbol $k$, the received signal vector writes:

$$
\mathbf{y}_{k}=f \sum_{i=1}^{N_{t}} \mathbf{h}_{i} x_{i}(k)+\mathbf{n}=f \mathbf{h}_{k}^{e q}+\mathbf{n}
$$

where $\mathbf{h}_{k}^{e q}$ is the equivalent channel vector received when sending the spatial symbol $k$. The detector has to estimate the index of antenna(s) that have been activated. The equation of the ML is:

$$
k=\operatorname{argmin}_{j}\left\|\mathbf{y}-f \mathbf{h}_{j}^{e q}\right\|^{2}
$$

2) Performance analysis: The Bit Error Probability (BEP) performance of the ESSK is given by the union bound equation:

$$
\mathcal{P}_{e}=\sum_{j} \sum_{k} \frac{\mathbf{d}\left(\mathbf{x}_{j}, \mathbf{x}_{k}\right)}{M} \mathcal{P}\left(\mathbf{x}_{j} \rightarrow \mathbf{x}_{k}\right)
$$

where $\mathcal{P}\left(\mathbf{x}_{k} \rightarrow \mathbf{x}_{j}\right)$ is the Pairwise Error Probability (PEP) and $d\left(\mathbf{x}_{k}, \mathbf{x}_{j}\right)$ is the hamming distance between two spatial symbols $\mathbf{x}_{k}$ and $\mathbf{x}_{j}$. Using Equation (4), the PEP conditioned on $\mathbf{H}$ is given as:

$$
\begin{aligned}
& \mathcal{P}\left(\mathbf{x}_{k} \rightarrow \mathbf{x}_{j} \mid \mathbf{H}\right)=\mathcal{P}\left(d_{k}>d_{j} \mid \mathbf{H}\right) \\
& =\mathcal{P}\left(\Re\left\{\mathbf{n}^{H}\left(\mathbf{h}_{j}^{e q}-\mathbf{h}_{k}^{e q}\right)\right\}>\frac{1}{2}\left\|\mathbf{h}_{j}^{e q}-\mathbf{h}_{k}^{e q}\right\|^{2} \mid \mathbf{H}\right) \\
& =Q(\sqrt{\kappa})
\end{aligned}
$$

where $d_{j}=\left\|\mathbf{y}-f \mathbf{h}_{j}^{e q}\right\|^{2}$ and $Q(x)$ is the Gaussian distribution function: $Q(x)=\int_{x}^{\infty} \frac{1}{2 \pi} e^{-\frac{t^{2}}{2}} d t$. We have also:

$$
\kappa=\frac{\left\|\mathbf{h}_{j}^{e q}-\mathbf{h}_{k}^{e q}\right\|^{2}}{2 \sigma_{n}^{2}}=\frac{\sum_{i=1}^{2 N_{r}} \alpha_{i}^{2}}{2 \sigma_{n}^{2}}
$$

where $\alpha_{i} \sim \mathcal{N}\left(0, \sigma_{\alpha}^{2}\right)$ with $\sigma_{\alpha}^{2}=\frac{f^{2} \mathbf{d}\left(\mathbf{x}_{j}, \mathbf{x}_{k}\right)}{4}$. The Average PEP for different channel realization is given by:

$$
\mathcal{P}\left(\mathbf{x}_{k} \rightarrow \mathbf{x}_{j}\right)=\mathbb{E}_{\mathbf{H}}\left[\mathcal{P}\left(\mathbf{x}_{k} \rightarrow \mathbf{x}_{j}\right)\right]=\int_{0}^{\infty} Q(\sqrt{u}) p_{\kappa}(u) d u,
$$

where $p_{\kappa}$ is the PDF of $\kappa$. The random variable $\kappa$ is chisquared distributed with $\mathrm{c}=2 N_{r}$ degrees of freedom, so we have:

$$
p_{\kappa}(u)=\frac{u^{\frac{c}{2}-1} \exp \left(-\frac{u}{2 \sigma_{\alpha}^{2}}\right)}{\left(2 \sigma_{\alpha}^{2}\right)^{\frac{c}{2}} \Gamma\left(\frac{c}{2}\right)},
$$

which has a closed form expression given in [14]:

$$
\mathcal{P}\left(\mathbf{x}_{k} \rightarrow \mathbf{x}_{j}\right)=\gamma_{\alpha}^{N_{r}} \sum_{k=1}^{N_{r}-1} C_{k}^{N_{r}-1+k}\left(1-\gamma_{\alpha}\right)^{k}
$$

where $\gamma_{\alpha}=\frac{1}{2}\left(1-\sqrt{\frac{\sigma_{\alpha}^{2}}{1+\sigma_{\alpha}^{2}}}\right)$.

\section{B. Equalization}

In the ERASK scheme, the channel estimation is used to create the precoding matrix used to transmit the spatial symbol. The same matrix can be used in uplink for the equalization, which is why it is interesting to evaluate the performance of the ESSK when using equalization.

1) Equalized Signal: The received signal $\mathbf{y}$ given in equation (1) is further equalized. Assuming perfect channel estimation at the receiver side, the equation of spatially equalized signals is:

$$
\mathbf{z}=f \mathbf{W H x}+\mathbf{W n}
$$

where $\mathbf{W} \in \mathbb{C}^{N_{t} \times N_{r}}$ is the equalization matrix. As a final step, a detector is used to estimate the spatial symbols from the equalized signal and demappes it to deliver the bit sequence corresponding to the mapping rule described above. The receiver uses the spatial equalization step to reconstruct the spatial symbol vector. The ZF equalization is employed, so:

$$
\mathbf{W}=\left(\mathbf{H}^{H} \mathbf{H}\right)^{-1} \mathbf{H}^{H} .
$$

Since the channel matrix is expected to be converted into an identity matrix after ZF equalization, any inter-antenna interference is removed at this step. To make it possible, the required number of antennas should however satisfy the 


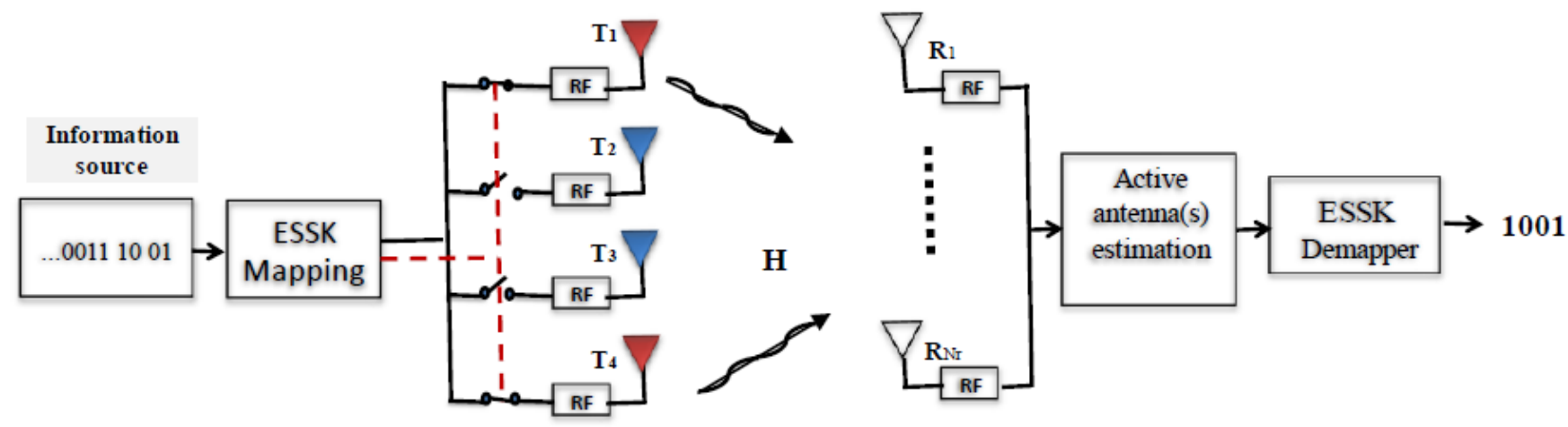

Figure 1. Block Diagram for Extended-SSK

constraint $N_{t} \leq N_{r}$ so that the matrix inversion remains possible. Assuming such a constraint is fulfilled, it is then straightforward to obtain the expression of the equalized signals:

$$
\mathbf{z}=f \mathbf{x}+\mu
$$

where $\mu=\mathbf{W n}$. Let $\mu_{i}$ be the $i$-th component of the vector $\mu \in \mathbb{C}^{N_{t} \times 1}$, and $w_{i, j}$ be the complex coefficient of matrix $\mathbf{W}$ in the $i$-th row and $j$-th column:

$$
\mu_{i}=\sum_{j=1}^{N_{r}} w_{i, j} \eta_{j} .
$$

To estimate the spatial symbols, the receiver should detect whether each transmit antenna is activated or not. From equation (13), the detector has to analyze the following set of signals:

$$
\forall i, \quad z_{i}= \begin{cases}f+\mu_{i} & \text { if } T_{i} \text { is activated } \\ \mu_{i} & \text { otherwise. }\end{cases}
$$

Since the ZF spatial equalization scheme is used, no interference appears between signal samples impinging at each receive antenna. In addition, since all activated antenna combinations are possible with the ESSK scheme, no correlation exists between the receive equalized signals. Consequently, the demodulation process can be led through an independent and parallel signal analysis per symbol, thereby simplifying the application of the maximum likelihood decision criterion. This is a strong asset of ESSK compared to conventional SM or GSM.

2) Equalized ML detection: The ML detection for the equalized signal can be reduced to a simple real part threshold as proofed in [13]:

$$
x_{i}= \begin{cases}0, & \text { if } \Re\left\{z_{i}\right\} \leq \nu, \\ 1, & \text { if } \Re\left\{z_{i}\right\} \geq \nu,\end{cases}
$$

where the optimal threshold is easily deduced from:

$$
\nu=\mathbb{E}\left\{\frac{\Re\left\{z_{i 0}\right\}+\Re\left\{z_{i 1}\right\}}{2}\right\}=\frac{f}{2}
$$

with $z_{i 0}$ (resp. $z_{i 1}$ ) the $i$-th element of equalized receive signal vector if the antenna $T_{i}$ wasn't active (resp. active). Note that such a threshold can in practice be estimated during a calibration phase using dedicated pilot symbols.

As proofed in [13], using such a threshold detector, the theoretical BEP can be expressed as follows:

$$
\mathcal{P}_{e}=\frac{1}{N_{t}} \sum_{i=1}^{N_{t}} Q\left(\sqrt{\frac{1}{N_{t}\left\|w_{i}\right\|^{2} \sigma_{n}^{2}}}\right) .
$$

\section{Simulation Results}

\section{A. Simulation descriptions}

The performance of the proposed system is evaluated through the measurement of the BER versus the Signal to Noise Ratio (SNR) that is equal to the ratio between the average transmit power level and noise level, i.e. $\mathrm{SNR}=\frac{\bar{P}_{t}}{\sigma_{n}^{2}}$. It is assumed that $\mathbf{H}$ is a MIMO flat fading channel matrix where $h_{j, i}$ are complex coefficients following an i.i.d. Rayleigh distribution. The power for each sub-channel is normalized:

$$
E\left[\left\|h_{j, i}\right\|^{2}\right]=1
$$

Finally, we consider that the channel response is perfectly known at the receiver, so that perfect $\mathrm{ZF}$ equalization is performed. Simulations are run by implementing a sufficient number of iterations for different channel realizations, and taking the mean value of the BER for each value of SNR.

\section{B. Theoretical Result Validation}

Analytical derivations of the BER are compared to the simulation results in Figure 2. An ESSK system with $N_{r}=8$ receive antennas and $N_{t}=4$ transmit antennas is considered. The performance is evaluated using the ML and EQ-ML detection. As evident from the obtained curves, theoretical results perfectly match simulation results. Also, it is seen that the ML without equalization outperforms the ML after equalization (EQ-ML).

\section{C. $M L$ vs EQ-ML performance}

In Figure 3, we compare the performance of $\mathrm{ML}$ and $\mathrm{EQ}-$ ML detection considering different configurations for $N_{r}$, and for the same $N_{t}=4$. ESSK systems with $N_{r}=4,8,16$ and 32 are simulated using the two detection methods. It is shown that ML detection outperforms the EQ-ML detection, but the 


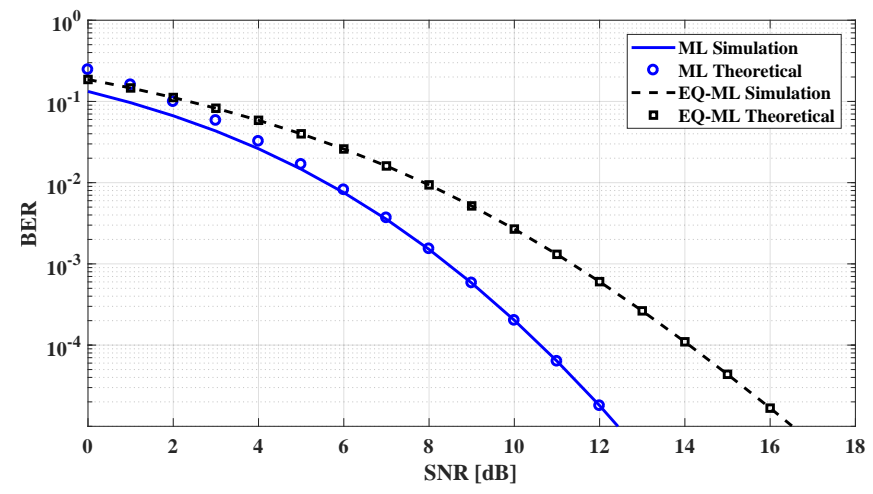

Figure 2. BER performance of $4 \times 8$ ESSK system, using ML and EQ-ML detection, theoretical and simulation comparison

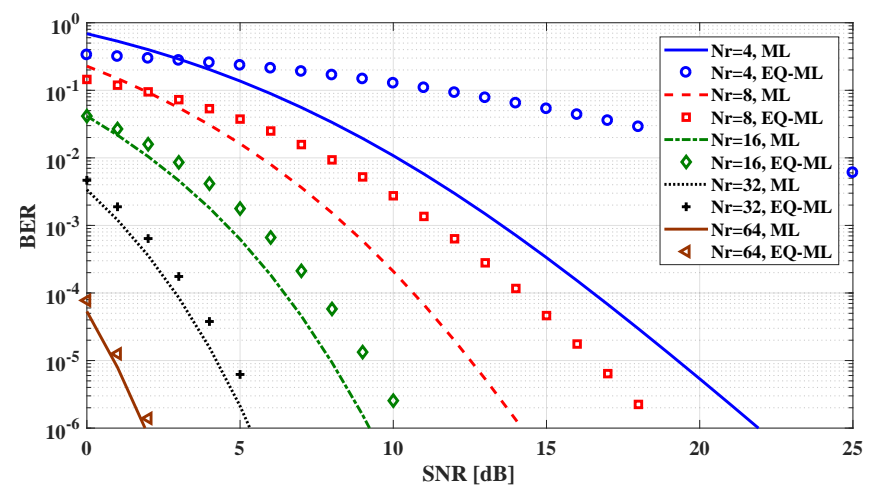

Figure 3. BER performance of $2 \times 8$ ESSK system, using ML and EQ-ML detection, theoretical and simulation comparison

more $N_{r}$ increases, the more the difference in performance decreases, and they become approximately the same for $N_{r}=$ 32 .

\section{Duality ESSK ERASK}

The uplink/ downlink duality in the MIMO wireless systems has been studied for different precoding- postcoding strategies [15]-[17]. Since the SM is a special kind of MIMO system, then a duality can be deduced between the transmit and receive SM.

In Figure ??, we provide the simulation results considering an ESSK system with $N_{r}=8$ receive antennas at the base station and 4 different transmit antenna array dimensions, namely $N_{t}=2,3$ and 4 at the device. On the other hand, in Figure 4, the simulation results considering an ERASK system with $N_{t}=8$ transmit antennas at the base station and 4 different receive antenna array dimensions, namely $N_{r}=2,3,4$ and 5 at the device. The two configurations seem to be the uplink and downlink transmission between the base station and a connected device. Note that for both scenarios, we are measuring the BER versus the SNR at the connected device. The performance is evaluated using the EQ-ML detection for ESSK and ML for ERASK. For

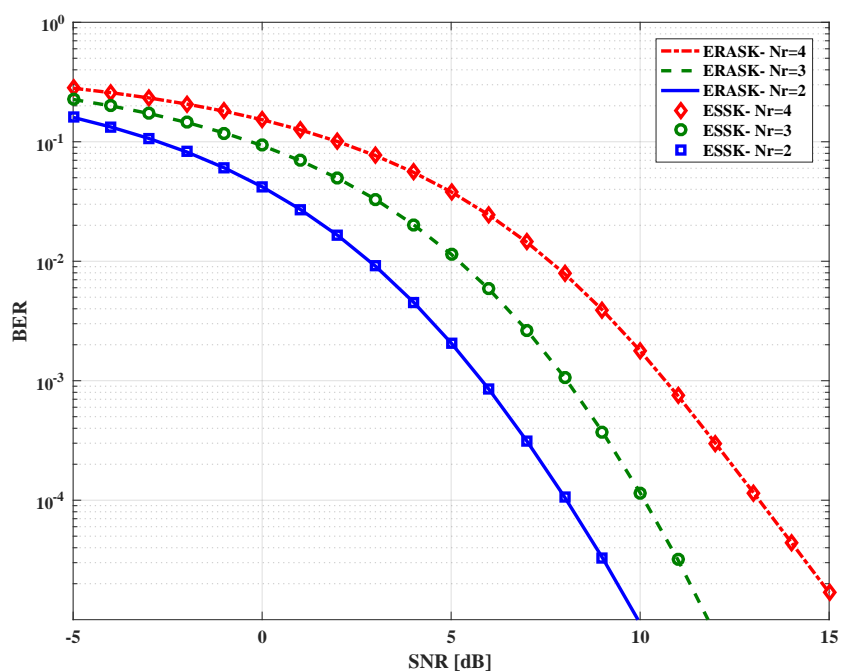

Figure 4. ERASK performance using ML detection with $N_{t}=8$ Theoretical and simulation comparison

both configurations, increasing the order of modulation, i.e. $N_{t}$ for ESSK or $N_{r}$ for ERASK, leads to a degradation in performance. Also, for the same order of modulation, we have approximately the same performance in the two transmissions, which proves the duality of transmission between ERASK and ESSK.

\section{SUMMARY}

In this paper, the concept of ESSK was presented, and we demonstrated how ESSK provides transmission of $m=N_{t}$ bits per spatial symbol duration. We also studied the ML detector, as well as the expected theoretical performance, and then the performance of the system when ZF equalization is performed. The ML detector outperforms the based equalizer detector, but results showed that the more the number of transmit antennas increases, the smaller the difference in performance is. Finally, we demonstrated the duality in performance between ERASK, and ESSK such that the based equalizer detector is implemented.

\section{ACKNOWLEDGMENT}

The authors would like to thank the SPATIAL MODULATION project funded by the French National Research Agency (ANR)

\section{REFERENCES}

[1] Y. A. Chau and S.-H. Yu, 'Space modulation on wireless fading channels', in Vehicular Technology Conference, 2001. VTC 2001 Fall. IEEE VTS 54th, IEEE, vol. 3, 2001, pp. 1668-1671.

[2] J. Jeganathan, A. Ghrayeb, L. Szczecinski, and A. Ceron, 'Space shift keying modulation for mimo channels', IEEE Transactions on Wireless Communications, vol. 8, no. 7, pp. 3692-3703, 2009. 
[3] M. Di Renzo, H. Haas, A. Ghrayeb, S. Sugiura, and L. Hanzo, 'Spatial modulation for generalized mimo: Challenges, opportunities, and implementation', Proceedings of the IEEE, vol. 102, no. 1, pp. 56-103, 2014.

[4] R. Y. Chang, S.-J. Lin, and W.-H. Chung, 'New space shift keying modulation with hamming code-aided constellation design', IEEE Wireless Communications Letters, vol. 1, no. 1, pp. 2-5, 2012.

[5] L.-L. Yang, 'Transmitter preprocessing aided spatial modulation for multiple-input multiple-output systems', in Vehicular Technology Conference (VTC Spring), 2011 IEEE 73rd, IEEE, 2011, pp. 1-5.

[6] A. Stavridis, S. Sinanovic, M. Di Renzo, and H. Haas, 'Transmit precoding for receive spatial modulation using imperfect channel knowledge', in Vehicular Technology Conference (VTC Spring), 2012 IEEE 75th, IEEE, 2012, pp. 1-5.

[7] D.-T. Phan-Huy and M. Hélard, 'Receive antenna shift keying for time reversal wireless communications', in 2012 IEEE International Conference on Communications (ICC), IEEE, 2012, pp. 4852-4856.

[8] A. Mokh, Y. Kokar, M. Hélard, and M. Crussière, 'Time reversal receive antenna shift keying on mimo los channel', in 2017 International Conference on Sensors, Networks, Smart and Emerging Technologies (SENSET), IEEE, 2017.

[9] R. Zhang, L.-L. Yang, and L. Hanzo, 'Generalised precoding aided spatial modulation', IEEE Transactions on Wireless Communications, vol. 12, no. 11, pp. 54345443, 2013.

[10] A. Mokh, M. Hélard, and M. Crussière, 'Extended receive antenna shift keying', in 2017 IEEE International Conference on Telecommunication (ICT), IEEE, 2017.

[11] A. Mokh, M. Crussière, and M. Hélard, 'Performance analysis of the maximum ratio transmission preprocessing for extended receive antenna shift keying', in 2017 International Symposium on Wireless Personal Multimedia Communications (WPMC), IEEE, 2017.

[12] — ' 'Performance analysis of extended rask under imperfect channel estimation and antenna correlation', in 2018 IEEE Wireless Communications and Networking Conference (WCNC), IEEE, 2018.

[13] A. Mokh, M. Hélard, and M. Crussière, 'Space shift keying modulations for low complexity internet-ofthings devices', in 2017 Global Communications Conference (GLOBECOM), IEEE, 2017.

[14] M.-S. Alouini and A. Goldsmith, 'A unified approach for calculating error rates of linearly modulated signals over generalized fading channels', in Communications, 1998. ICC 98. Conference Record. 1998 IEEE International Conference on, IEEE, vol. 1, 1998, pp. 459-464.

[15] T. Endeshaw, B. K. Chalise, and L. Vandendorpe, 'Mse uplink-downlink duality of mimo systems under imperfect csi', in Computational Advances in Multi-Sensor Adaptive Processing (CAMSAP), 2009 3rd IEEE International Workshop on, IEEE, 2009, pp. 384-387.
[16] S. Shi, M. Schubert, and H. Boche, 'Downlink mmse transceiver optimization for multiuser mimo systems: Duality and sum-mse minimization', IEEE Transactions on Signal Processing, vol. 55, no. 11, pp. 54365446, 2007.

[17] M. Ding and S. D. Blostein, 'Uplink-downlink duality in normalized mse or sinr under imperfect channel knowledge', in Global Telecommunications Conference, 2007. GLOBECOM'07. IEEE, IEEE, 2007, pp. 3786-3790. 